\title{
Growth of a progesterone receptor-positive meningioma in a female patient with congenital adrenal hyperplasia
}

\author{
T O'Shea', R K Crowley', M Farrell², S MacNally³, P Govender ${ }^{4}$, J Feeney4, J Gibney ${ }^{1}$ \\ and $M$ Sherlock \\ 1Department of Endocrinology, Adelaide and Meath Hospitals, incorporating the National Children's \\ Hospital, Tallaght, Dublin, Ireland, Departments of ${ }^{2}$ Neuropathology, ${ }^{3}$ Neurosurgery, National \\ Neurosciences Centre, Beaumont Hospital, Beaumont, Dublin, Ireland, and 4Department of Radiology, \\ Adelaide and Meath Hospitals, incorporating the National Children's Hospital, Tallaght, Dublin, Ireland
}

Correspondence should be addressed to M Sherlock Email

mark.sherlock@amnch.ie

\section{Summary}

Meningioma growth has been previously described in patients receiving oestrogen/progestogen therapy. We describe the clinical, radiological, biochemical and pathologic findings in a 45-year-old woman with congenital adrenal hyperplasia secondary to a defect in the 21-hydroxylase enzyme who had chronic poor adherence to glucocorticoid therapy with consequent virilisation. The patient presented with a frontal headache and marked right-sided proptosis. Laboratory findings demonstrated androgen excess with a testosterone of $18.1 \mathrm{nmol} / \mathrm{L}(0-1.5 \mathrm{nmol})$ and 17 -Hydroxyprogesterone $>180 \mathrm{nmol} / \mathrm{L}(<6.5 \mathrm{nmol} / \mathrm{L})$. CT abdomen was performed as the patient complained of rapid-onset increasing abdominal girth and revealed bilateral large adrenal myelolipomata. MRI brain revealed a large meningioma involving the right sphenoid wing with anterior displacement of the right eye and associated bony destruction. Surgical debulking of the meningioma was performed and histology demonstrated a meningioma, which stained positive for the progesterone receptor. Growth of meningioma has been described in postmenopausal women receiving hormone replacement therapy, in women receiving contraceptive therapy and in transsexual patients undergoing therapy with highdose oestrogen and progestogens. Progesterone receptor positivity has been described previously in meningiomas. 17-Hydroxyprogesterone is elevated in $\mathrm{CAH}$ and has affinity and biological activity at the progesterone receptor. Therefore, we hypothesise that patients who have long-standing increased adrenal androgen precursor concentrations may be at risk of meningioma growth.

\section{Learning points:}

- Patients with long-standing CAH (particularly if not optimally controlled) may present with other complications, which may be related to long-standing elevated androgen or decreased glucocorticoid levels.

- Chronic poor control of CAH is associated with adrenal myelolipoma and adrenal rest tissue tumours.

- Meningiomas are sensitive to endocrine stimuli including progesterone, oestrogen and androgens as they express the relevant receptors.

\section{Background}

Congenital adrenal hyperplasia (CAH) is the most common adrenal disorder diagnosed in children and is secondary to a defect in the 21-hydroxylase enzyme in more than $95 \%$ of cases. Virilisation of affected female children occurs in both the salt-losing and simple virilising forms of CAH. In normal adrenal steroidogenesis, 
ACTH promotes the production of pregnenolone from cholesterol, which is then converted to progesterone and 17-hydroxyprogesterone (17OHP) and cortisol. In the absence of 21-hydroxylase enzyme activity, production of cortisol (and to a varying extent aldosterone) is reduced, leading to increased ACTH secretion due to a decrease in negative feedback by cortisol at the level of the pituitary and hypothalamus. In 21 hydroxylase deficiency, steroid precursors are diverted into androgen synthesis pathways leading to testosterone, androstenedione and 17OHP

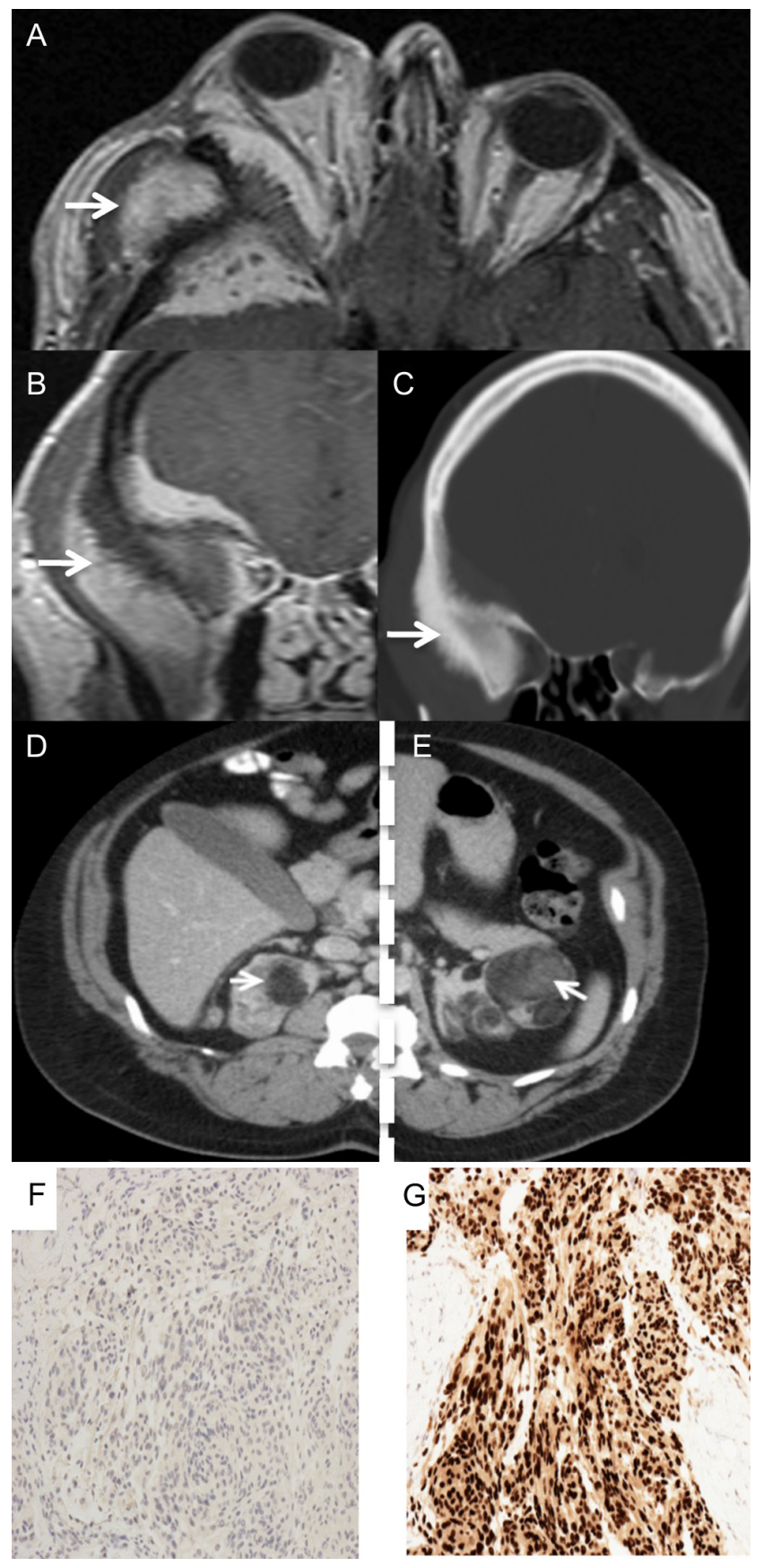

excess. The major treatment goals in adults with $\mathrm{CAH}$ include avoidance of adrenal crisis, prevention of adrenal and gonadal hyperplasia and neoplasia, the prevention of long-term consequences of adrenal replacement therapies and optimisation of fertility (if required).

Meningiomas are the most common primary brain tumours in adults (1). The higher prevalence of meningiomas in women suggests a link between sex steroids and meningiomas (1). Meningiomas commonly express the progesterone receptor (PR) and less commonly, oestrogen and androgen receptors (1).

\section{Case presentation}

\section{Case report}

A 45-year-old woman was diagnosed with CAH secondary to a defect in 21-hydroxylase (non-salt wasting, simple virilising) in childhood, at which time she presented with premature adrenarche and virilisation. Glucocorticoid therapy was initiated, but adherence throughout childhood and adolescence was suboptimal. Menarche was never achieved, and the patient was lost to follow-up. At the age of 45 years, the patient was hospitalised because of severe frontal headaches, increasing in intensity over the preceding three years. Her height was $148 \mathrm{~cm}$; body mass index $27 \mathrm{~kg} / \mathrm{m}^{2}$; and cardiovascular, respiratory and gastrointestinal system examinations were unremarkable. On examination, the patient was virilised. Right-sided proptosis, but no diplopia, was evident but visual acuity was preserved, and focal neurological deficits were not apparent.

Laboratory investigations confirmed significant hyperandrogenaemia with testosterone $18.1 \mathrm{nmol} / \mathrm{L}$ (reference range $0-1.5 \mathrm{nmol} / \mathrm{L}$ ) and 17-Hydroxyprogesterone (17-OHP) levels greater than the upper limit

\section{Figure 1}

(A) Axial gadolinium contrast-enhanced MRI demonstrating an intensely enhancing $4.3 \times 4.6 \mathrm{~cm}$ soft tissue mass centred upon the right greater wing of sphenoid (arrow), expanding into the middle cranial fossa, lateral orbit and infra-temporal fossa, with a marked right proptosis. There is a spiculated periosteal reaction involving the lateral orbital wall. (B) Coronal contrast-enhanced MRI demonstrates an enhancing soft tissue mass centred upon the right greater wing of sphenoid, measuring $6.0 \mathrm{~cm}$ in craniocaudal extent, with the involvement of the squamous part of the temporal bone (arrow). An extra-axial intracranial component shows typical features of a meningioma, with homogenous intense enhancement and a dural tail. (C) Coronal CT head (bone windows) showing the spiculated 'sunray' periosteal reaction of the right greater wing of sphenoid (arrow). (D) and (E) - CT abdomen showing large bilateral adrenal myelolipomata up to $4.5 \mathrm{~cm}$ (arrows). (F) and (G) histology from meningioma specimen showing negative oestrogen receptor expression (F) and positive progesterone receptor expression (G). 
of detection of the assay at $>180 \mathrm{nmol} / \mathrm{L}$ (reference range $<6.5 \mathrm{nmol} / \mathrm{L}$ ). ACTH concentration for this patient was $519 \mathrm{pg} / \mathrm{mL}$ (reference range $0-46 \mathrm{pg} / \mathrm{mL}$ ). CT and MRI imaging (Fig. 1A, B and C) revealed a solid enhancing mass (measuring $4.3 \times 4.6 \mathrm{~cm}$ ) centred upon the greater wing of the right sphenoid bone, expanding into the middle cranial fossa, orbit and infra-temporal fossa associated with an aggressive periosteal reaction and a dural tail. There was significant mass effect on the right orbit, with proptosis and stretching of the right lateral rectus muscle and optic nerve, but no evidence of increased intracranial pressure from a radiological or clinical perspective. She also complained of an increase in abdominal girth, and CT imaging of the abdomen (Fig. 1D and E) revealed bilateral adrenal masses characteristic of adrenal myelolipoma in keeping with radiological evidence of chronic ACTH hyperstimulation (2), but no other pathology was evident.

The patient underwent debulking neurosurgery with stress dose intravenous hydrocortisone as perioperative cover. Histology demonstrated a meningioma (WHO Grade 1), which stained strongly positive for progesterone receptors and negative for oestrogen receptors (Fig. 1F and G). Improved adherence with glucocorticoid therapy was advised.

Periodically, glucocorticoid dosage was increased as high as $35 \mathrm{mg}$ of hydrocortisone/24h. On glucocorticoid therapy, the patients' most recent androstenedione concentrations were $>30.0 \mathrm{nmol} / \mathrm{L}$ (reference range 1.7-16.4), $17-\mathrm{OHP} 705 \mathrm{nmol} / \mathrm{L}$ (reference range $<6.5 \mathrm{nmol} / \mathrm{L}$ ) and testosterone $9.5 \mathrm{nmol} / \mathrm{L}$ (reference range $0-1.5 \mathrm{nmol} / \mathrm{L}$ ). Plasma renin activity was consistently within the normal range with the last assessment being $3.7 \mathrm{ng} / \mathrm{mL} / \mathrm{h}$ (erect reference range 1.0-4.2). Plasma aldosterone concentration was also normal on the last assessment $794.7 \mathrm{pmol} / \mathrm{L}$ (erect reference range 194-970 pmol/L). Renal profile and electrolytes were normal on the last review. The patient opted for a lower dose of glucocorticoid replacement of $15 \mathrm{mg}$ of hydrocortisone $/ 24 \mathrm{~h}$ as she felt higher doses were contributing to weight gain.

\section{Investigation}

Laboratory investigations confirmed significant hyperandrogenaemia with testosterone $18.1 \mathrm{nmol} / \mathrm{L}$ (reference range $0-1.5 \mathrm{nmol} / \mathrm{L}$ ), androstenedione $>30 \mathrm{nmol} / \mathrm{L}$ (reference range 1.7-16.4) and 17-Hydroxyprogesterone (17-OHP) levels greater than the upper limit of detection of the assay at $>180 \mathrm{nmol} / \mathrm{L}$ (reference range $<6.5 \mathrm{nmol} / \mathrm{L}$ ). ACTH concentration for this patient was $519 \mathrm{pg} / \mathrm{mL}$ (reference range $0-46 \mathrm{pg} / \mathrm{mL}$ ). CT and MRI imaging (Fig. 1A, B and C) revealed a solid enhancing mass centred upon the greater wing of the right sphenoid bone, expanding into the middle cranial fossa, orbit and infra-temporal fossa associated with an aggressive periosteal reaction and a dural tail. There was significant mass effect on the right orbit, with proptosis and stretching of the right lateral rectus muscle and optic nerve. She also complained of an increase in abdominal girth, and CT imaging of the abdomen (Fig. 1D and E) revealed bilateral adrenal masses characteristic of adrenal myelolipoma in keeping with radiological evidence of chronic ACTH hyperstimulation (2), but no other pathology was evident.

\section{Treatment}

The patient underwent debulking neurosurgery, and post-operatively, the tumour dimensions had decreased from $4.3 \times 4.6 \mathrm{~cm}$ to $3.5 \times 2.1 \mathrm{~cm}$. Histology demonstrated a meningioma (WHO Grade 1), which stained strongly positive for progesterone receptors and negative for oestrogen receptors (Fig. 1F and G).

\section{Outcome and follow-up}

Improved adherence with glucocorticoid therapy was advised. Periodically, glucocorticoid dosage was increased as high as $35 \mathrm{mg}$ of hydrocortisone/24 h; however, during subsequent follow-up, her 17OHP and androstenedione levels remained markedly elevated. The patient opted for a lower dose of glucocorticoid replacement of $15 \mathrm{mg}$ of hydrocortisone $/ 24 \mathrm{~h}$ as she felt higher doses were contributing to weight gain. The meningioma was stable with no clinically significant growth after debulking after 4-year follow-up, and the patient was under neurosurgical follow-up who deemed there was no indication for further surgical intervention at present.

\section{Discussion}

Meningiomas occur twice as frequently in women as in men. This gender difference has been attributed to the expression of sex steroid receptors by meningiomas; approximately $68 \%$ of meningiomas express receptors for progesterone (PR), whereas a smaller number express oestrogen receptors and androgen receptors (1).

To date, there have been two reported cases of growth of PR-expressing meningiomas in patients with 
congenital adrenal hyperplasia $(3,4)$. Our patient also has evidence of bilateral giant adrenal myelolipomas, which is likely due to chronic ACTH hyperstimulation. These findings have never been reported in combination and suggest a link between poorly controlled CAH and development of these tumours. In patients with $\mathrm{CAH}$ and inadequate glucocorticoid replacement, stimulation of meningioma growth may possibly be influenced by elevated adrenocorticotrophin hormone (ACTH) or to elevated androgens and their precursors. Occurrence of these 2 meningiomas in $\mathrm{CAH}$ and further reports of meningioma growth in postmenopausal women receiving post-menopausal hormone replacement therapy, longacting reversible contraceptives and pregnancy, suggest that progesterone or its precursors may play a role in the growth and pathogenesis of meningiomas; however, the data are conflicting. Indeed, epidemiological studies of factors associated with increased prevalence and accelerated growth of meningiomas have confirmed a link with exogenous sex steroids but cannot prove causality $(5,6)$.

In our patient, 17OHP concentrations were significantly elevated and most likely had been so for some time as evident by the bilateral adrenal myelolipomas and clinical features. Adrenal myelolipomas are rare benign adrenal tumours, which are composed largely of mature fat and can be identified by this characteristic on imaging studies (2). They have increasingly been reported in patients with poorly controlled $\mathrm{CAH}$ and have been hypothesised to occur as a result of chronic stimulation of the adrenal gland by high levels of adrenocorticotropic hormone (ACTH).

$17 \mathrm{OHP}$ has been shown to have affinity for and bind to the progesterone receptor (varying binding affinities have been reported) (7). To our knowledge, the binding affinity of $17 \mathrm{OHP}$ to progesterone receptors in situations of supraphysiological levels of 17OHP in female patients with $\mathrm{CAH}$ has not been addressed. Estrone and testosterone also interact with human PR (8), both of which are also elevated in patients with poorly controlled CAH. Small in vitro studies have shown some growth of meningioma cells in the presence of progesterone, perhaps by the modulation of epidermal growth factor (9). In recent years, it has been increasingly recognised that adult patients with $\mathrm{CAH}$ are underrepresented in adult endocrinology clinics and are known to face multiple problems in adulthood including reduced fertility rates, greater rates of metabolic disease and osteopenia and subjectively reduced quality of life (10).
The growth of meningioma in our patient with $\mathrm{CAH}$ is an association (given the relative frequency of $\mathrm{CAH}$ and meningioma) and therefore routine imaging is not recommended in all patients with $\mathrm{CAH}$. We hypothesise that the prolonged supraphysiologic exposure to $17 \mathrm{OHP}$ levels in our patient reported here may have accelerated meningioma growth by stimulation of the PR. Whether patients with poorly controlled CAH with elevated levels of $17 \mathrm{OHP}$ are at greater risk for meningioma growth or whether 17OHP accelerates meningioma growth needs further study. Similarly, whether reducing $17 \mathrm{OHP}$ will have an effect on tumour growth requires further study.

\section{Declaration of interest}

The authors declare that there is no conflict of interest that could be perceived as prejudicing the impartiality of the research reported.

\section{Funding}

This research did not receive any specific grant from any funding agency in the public, commercial or not-for-profit sector.

Patient consent

The patient has given informed written consent for the publication of this manuscript and has reviewed the manuscript prior to submission.

\section{Author contribution statement}

All authors were involved in writing and reviewing the manuscript. T O S, R K C, J G and M S were involved in the endocrine clinical management of patient. P G and J F were involved in radiological investigations. S Mc N and $\mathrm{M} \mathrm{F}$ were involved in the neurosurgical aspects of the case.

\section{References}

1 Wiemels J, Wrensch M \& Claus EB 2010 Epidemiology and etiology of meningioma. Journals of Neurooncology 99 307-314. (doi:10.1007/ s11060-010-0386-3)

2 Kok HK, Sherlock M, Healy NA, Doody O, Govender P \& Torreggiani WC 2015 Imaging features of poorly controlled congenital adrenal hyperplasia in adults. British Journal of Radiology 88 20150352. (doi:10.1259/bjr.20150352)

3 Heijboer AC, Netelenbos JC \& Blankenstein MA 2009 Meningioma in untreated congenital adrenal hyperplasia: a relationship? Journals of Neurooncology 92 223-225. (doi:10.1007/s11060-008-9751-x)

4 Li X \& Zhao J 2009 Intracranial meningiomas of childhood and adolescence: report of 34 cases with follow-up. Child's Nervous System 25 1411-1417. (doi:10.1007/s00381-009-0949-9)

5 Hatch EE, Linet MS, Zhang J, Fine HA, Shapiro WR, Selker RG, Black PM \& Inskip PD 2005 Reproductive and hormonal factors and risk of brain tumors in adult females. International Journal of Cancer 114 797-805. (doi:10.1002/ijc.20776)

6 Jhawar BS, Fuchs CS, Colditz GA \& Stampfer MJ 2003 Sex steroid hormone exposures and risk for meningioma. Journal of Neurosurgery 99 848-853. (doi:10.3171/jns.2003.99.5.0848) 
7 Attardi BJ, Zeleznik A, Simhan H, Chiao JP, Mattison DR \& Caritis SN 2007 Comparison of progesterone and glucocorticoid receptor binding and stimulation of gene expression by progesterone, 17-alpha hydroxyprogesterone caproate, and related progestins. American Journal of Obstetrics and Gynecology 197 599.e591-e597. (doi:10.1016/j. ajog.2007.05.024)

8 Hasan TN, B LG, Masoodi TA, Shafi G, Alshatwi AA \& Sivashanmugham P 2011 Affinity of estrogens for human progesterone receptor A and B monomers and risk of breast cancer: a comparative molecular modeling study. Advances and Applications in Bioinformatics and Chemistry 4 29-36. (doi:10.2147/AABC. S17371)

9 Koper JW \& Lamberts SW 1994 Meningiomas, epidermal growth factor and progesterone. Human Reproduction 9 (Supplement 1) 190-194. (doi:10.1093/humrep/9.suppl_1.190)

10 Arlt W, Willis DS, Wild SH, Krone N, Doherty EJ, Hahner S, Han TS, Carroll PV, Conway GS, Rees DA, et al. 2010 Health status of adults with congenital adrenal hyperplasia: a cohort study of 203 patients. Journal of Clinical Endocrinology and Metabolism 95 5110-5121. (doi:10.1210/jc.2010-0917)

Received in final form 20 September 2016

Accepted 25 October 2016 